\title{
Retrospective analysis and modifications of retractor systems for anterior odontoid screw fixation
}

\author{
Stan G. Shalayev, M.D., In Ki Mun, Ph.D., Gregory S. Mallek, Sylvain Palmer, M.D., \\ Allan D. Levi, M.D., Ph.D., Todd M. Lasner, M.D., and Allen B. Kantrowitz, M.D.
}

Neurosurgery Associates of Miami Beach, Florida; Jackson Memorial Hospital, Miami, Florida; Medtronic Sofamor Danek, Memphis, Tennessee; and Mission Hospital Regional Medical Center, Mission Viejo, California

\begin{abstract}
The authors present an in-depth retrospective analysis of retractor systems for anterior odontoid screw fixation They discuss various modifications and innovations of such systems and describe their own tubular retractor system, in which a beveled end conformal to the ventral surface of the vertebral column at the C2-3 level is introduced together with an incorporated light source. This new retractor system allows optimal anatomical exposure for controlled odontoid screw placement with improved protection of surrounding vital structures.
\end{abstract}

\section{KEY WORDS • retractor system • odontoid fracture • anterior screw fixation • tubular retractor}

Click here to view Video Clip 1 with sound. Retrospective analysis and modifications of a tubular retractor systems for anterior odontoid screw fixation. Allen B. Kantrowitz, M.D.

Anterior screw fixation of the dens has entered the surgical armamentarium for the management of odontoid fractures, a subject of surgical controversy. ${ }^{2,6,7,11-13} \mathrm{Com}-$ pared with other therapeutic options that are available, including external halo fixation and posterior cervical fusion, anterior screw fixation of the dens can potentially lower the morbidity rate in patients. ${ }^{4,8,14,15,17}$ Anterior screw fixation of the dens was initially described independently by Nakashimi and Boehler in the early 1980s. Extensive series of long-term studies undertaken by Aebi and colleagues ${ }^{1}$ and by Apfelbaum, et al., ${ }^{3}$ demonstrated an excellent clinical feasibility of the approach by providing documentation of a fusion rate of $88 \%$ in acute cases and $25 \%$ in chronic cases of nonunion of the dens. A retrospective analysis of approaches for odontoid fracture fixation revealed that a rigid-tunnel type of construct is the most beneficial solution for this intervention as long as soft tissues in the high cervical segments are protected while the instrumentation is being placed. Toward that end, Apfelbaum introduced a modification of a conventional anterior cervical retractor. In his design, the conventional transverse anterior cervical retractor is augmented by a detachable third blade that is capable of supporting the posterior wall of the pharynx. A toothed tube is subsequently driven into the ventral surface of $\mathrm{C}-3$ to protect local tissues further from the rotating surgical tools.

Abbreviation used in this paper: $\mathrm{CT}=$ computerized tomography.
Hott, et al., ${ }^{9}$ have recently described the use of the METRx retractor ${ }^{5,10}$ (Medtronic Sofamor Danek, Memphis, TN) as a retractor system for this surgery, to avoid mobilization of the longus colli muscle by stabilizing the retractor with a bedside rail.

In this paper we present a further modification of the METRx system, in which the tubular retractor has been optimized to permit visualization and retraction along the anticipated trajectory that is required for placement of the odontoid screw. A light source has also been incorporated in the new design.

\section{DESCRIPTION OF THE DEVICE INNOVATIONS AND THE METHOD OF USE}

We have modified a METRx tubular retractor by introducing a beveled end that is conformal to the ventral surface of the vertebral column at the $\mathrm{C} 2-3$ level (Figs. 1 and 2).

The angle of the beveled edge corresponds to the angle between the ventral surface of C-2 and the anticipated trajectory of the vertebral body screw. This bevel allows a close conformation between the open end of the tubular retractor and the ventral surface of the vertebral column, thereby excluding mobile soft tissues from the surgical field. This modified tube can still be supported by the bedside rail in a conventional fashion. The retractor can be positioned conventionally, as described by Hott and associates. ${ }^{9}$ A concentric light source can be placed within the tube, thereby greatly improving the surgeon's view (Figs. 1 right and 3). 

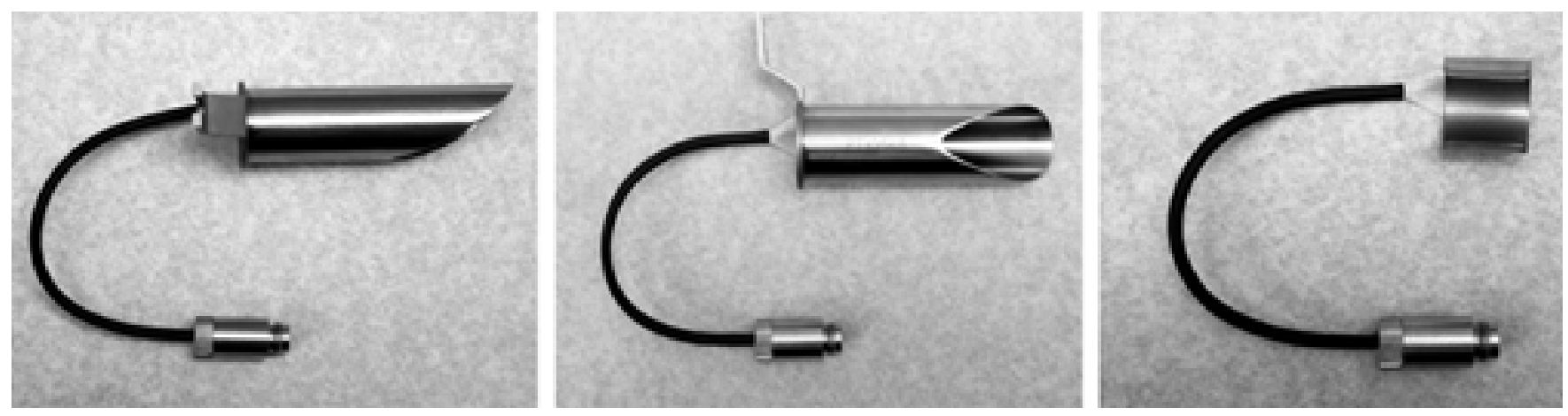

Fig. 1. Photographs showing the modified tubular retractor with an incorporated light source. Left: Lateral view. Center: Base view. Right: The light source.

\section{ILLUSTRATIVE CASE}

History. This 84-year-old woman fell and sustained direct trauma to her upper forehead region. She complained of neck pain and stiffness, and was brought to the emergency department at our institution.

Examination. A physical examination revealed no neurological deficits. There was severe neck tenderness in response to palpation in the posterior upper cervical area. Evaluation of the patient included CT scanning of the brain, which revealed no evidence of hemorrhage, and CT scanning of the cervical spine, which demonstrated a Type II dens fracture due to a structural failure that had been caused by a hyperextension mechanism of injury (Fig. 4).

Operation. The patient and her family refused a trial placement of a Halo orthosis; anterior screw fixation was accepted as a treatment option. The patient was placed on a radiolucent operating table. A radiolucent mouth gag was used to maximize the open-mouth view of the dens and a biplanar fluoroscope was positioned. To identify any potential interference between the chest or mandible and the surgical instrumentation, an extended K-wire was placed on the patient's shoulder, close to the neck along the anterior chest wall. This was done in a manner to simulate the anticipated trajectory of screw placement

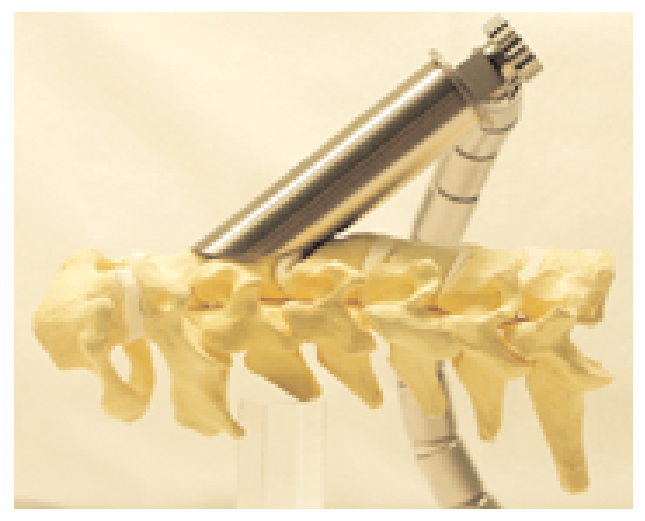

Fig. 2. Photograph showing a model of a spinal cervical segment and the retractor as it should be positioned for screw placement. The appropriate trajectory should be confirmed using biplanar fluoroscopy. in the lateral fluoroscopic view, thereby confirming the feasibility of the planned trajectory. A right-sided transverse skin incision was made at the point where the surgical trajectory was anticipated to intersect the skin surface. Cosmetic issues were taken into consideration as minor adjustments were made in the details of the incision. Conventional anterior cervical dissection techniques were used to enter the prevertebral space. ${ }^{16}$

The tube retractor was stabilized via the bedside rail, rather than by placement of transverse cervical blades, and thus the longus colli muscle in the middle cervical region was left undisturbed. Using a conventional technique, the prevertebral anatomical plane was dissected bluntly and the prevertebral space was enlarged to accommodate the retractor tube at the level of the $\mathrm{C} 2-3$ disc space. Using biplanar fluoroscopy for confirmation, the modified beveled tubular retractor was placed precisely to follow the surgical trajectory and straddle the region adjacent to the C2-3 disc space. The concentric light source was positioned and we were able to confirm visually that we had attained excellent protection of adjacent mobile soft tissues and had straddled the transverse curvature of the ventral surface of the vertebral column. Following standard procedures, a small midline osteotomy was performed in the corpus of C-3 to expose the cartilaginous endplate of

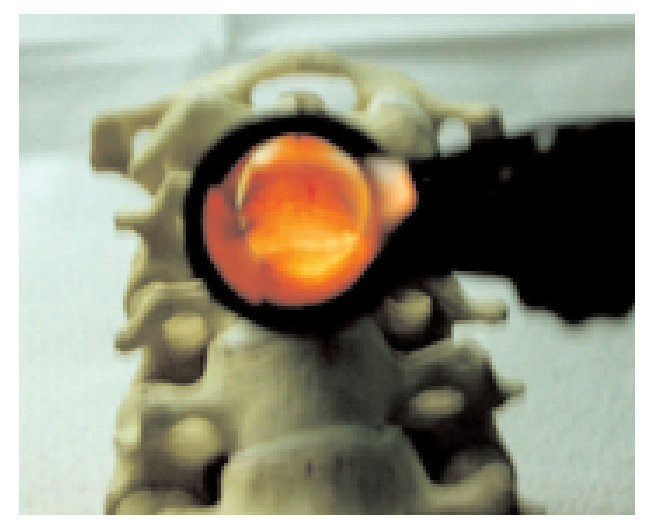

Fig. 3. Surgeon's view as the C2-3 disc is straddled by the retractor, with the incorporated light source. 


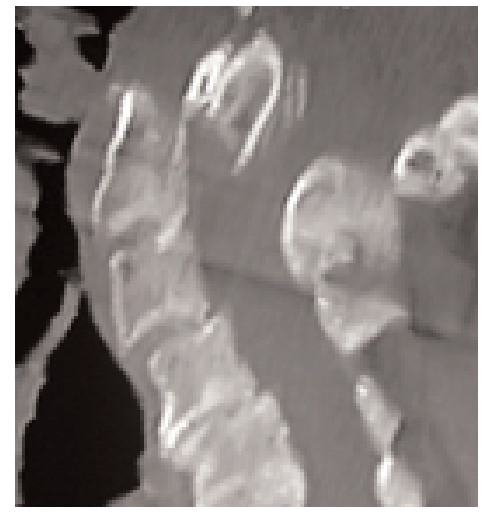

Fig. 4. A CT scan demonstrating a Type II odontoid fracture due to a structural failure that had been caused by a hyperextension mechanism of injury.

the $\mathrm{C} 2-3$ disc, after which the disc material was removed to visualize the inferior endplate of C-2. Under the control of biplanar fluoroscopy, a K-wire was placed within the corpus of C-2 and, with reduction of the fracture, the wire was advanced across the fracture zone into the dens. The K-wire was followed by a cannulated drill and, subsequently, by a cannulated titanium screw with the intermittent aid of biplanar fluoroscopy (Fig. 5).

Postoperative Course. The patient had an uneventful recovery. Postoperative CT scans demonstrated an adequate reduction of the fracture and appropriate screw placement (Figs. 6 and 7).

\section{DISCUSSION}

One challenge to the surgeon who performs an anterior screw fixation of the dens is to maintain adequate protection of the posterior pharyngeal wall during the sequential steps of the procedure. Another is that an ectatic carotid artery can prolapse into the surgeon's field of view. These vital structures and other surrounding tissues are at risk during the procedure, particularly when the surgeon is using rotating equipment. Hand-held retractors are awkward to place and difficult for the surgical assistant to maintain
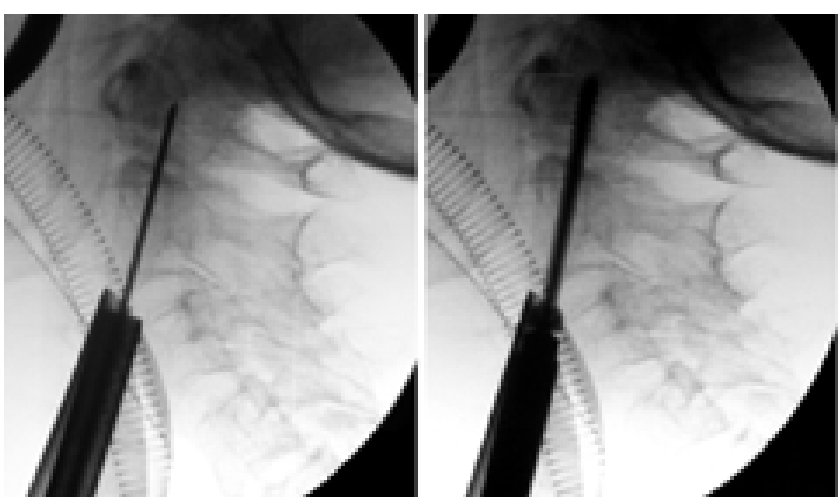

Fig. 5. Intraoperative fluoroscopic images obtained after fracture reduction and advancement of the K-wire (left) and after advancement of the cannulated screw (right).

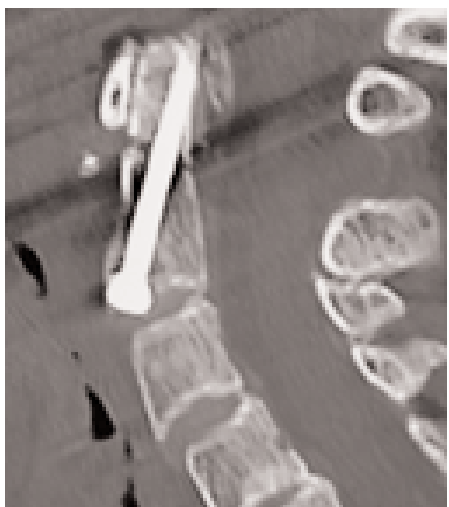

Fig. 6. Sagittal CT reconstruction demonstrating fracture reduction and screw fixation. Note the congruence between the geometry of the beveled tubular retractor and the trajectory of the screw placement.

in the proper position throughout the duration of the procedure. The limited view of the surgical field compounds the assistant's difficulties. The Apfelbaum retractor represents an important advancement in the management of these cases and provides a stable elevation and protection of the posterior pharyngeal wall. Highly mobile soft tissues might prolapse into the surgical field from around the sides of the pharyngeal retractor blade. Hott, et al., ${ }^{9}$ and Sonntag and colleagues, ${ }^{16}$ in adapting the METRx retractor for anterior screw fixation of the dens, have obviated the need for mobilization of the longus colli muscle to accommodate the transverse blades of the Apfelbaum retractor. Proper placement of the standard METRx tube is determined by the interference between the dorsal lip of the retractor tube and the ventral surface of the C-3 corpus. Mobile soft-tissue structures neighboring the gap that exists beyond the limit of the tube retractor remain at risk.

In an effort to maximize protection of adjacent soft tissues during the operation, we have identified several design considerations. Of highest importance, the retractor needs to protect the posterior wall of the pharynx and to exclude ectatic segments of the high cervical carotid arteries from the surgical field. In addition, the design should capture the advantage identified by Hott, et al., ${ }^{9}$ of avoid-

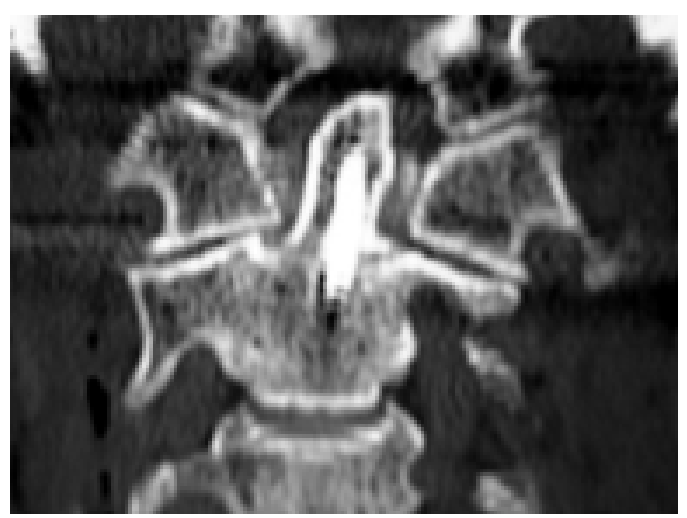

Fig. 7. Coronal CT scan demonstrating accurate cannulation of the dens. 
ance of unnecessary dissection of the longus colli muscle merely to serve as an anchor for the retractor system. Additionally, one would anticipate that a design that conforms to the transverse curvature of the ventral surface of the vertebral column would have less tendency to migrate during surgery. A light source could advantageously be included if it leaves open the surgeon's view.

These considerations are satisfied with the use of the modified METRx tubular retractor with the beveled edge. The angle of the beveled edge corresponds to the angle between the ventral surface of C-2 and the anticipated trajectory of the vertebral body screw. This bevel allows close conformation between the open end of the tubular retractor and the ventral surface of the vertebral column, thereby excluding mobile soft tissues from the surgical field. This modified tube can still be supported by the bedside rail in a conventional fashion.

We have found that this beveled retractor eases the technical difficulty of retraction of soft tissues when anterior screw fixation of the dens is performed. Moreover, the assistant can be reassigned to aid with the reduction of the fracture at the moment the K-wire is advanced. The system is simple to use and generally available.

Click here to view Video Clip 2 with sound. Modified tubular retractor system for odontoid screw fixation. Case presentation by Allan D. Levi, M.D., Ph.D.

\section{Disclosure}

Gregory Mallek is an employee of Medtronic Sofamor Danek. Dr. Sylvain Palmer is a consultant to that company and a participant in its speaker's bureau. The other authors have no financial interest in the company.

\section{References}

1. Aebi M, Etter C, Coscia M: Fractures of the odontoid process. Treatment with anterior screw fixation. Spine 14:1065-1070, 1989

2. Anderson LD, D'Alonzo RT: Fractures of the odontoid process of the axis. J Bone Joint Surg 56:1663-1674, 1974

3. Apfelbaum RI, Lonser RR, Veres R, et al: Direct anterior screw fixation for recent and remote odontoid fractures. J Neurosurg (Spine 2) 93:227-236, 2000
4. Etter C, Coscia M, Jaberg H, et al: Direct anterior fixation of dens fractures with a cannulated screw system. Spine 16 (Suppl 3):S25-S32, 1991

5. Foley KT, Smith M: Microendoscopic discectomy. Techn Neurosurg 3:301-307, 1997

6. Fujii E, Kobayashi K, Hirabayashi K: Treatment in fractures of the odontoid process. Spine 13:604-609, 1988

7. Haid RW Jr, Subach BR, McLaughlin MR, et al: C1-C2 transarticular screw fixation for atlantoaxial instability: a 6-year experience. Neurosurgery 49:65-70, 2001

8. Hashizume H, Kawakami M, Kawai M, et al: A clinical case of endoscopically assisted anterior screw fixation for the type II odontoid fracture. Spine 28:E102-E105, 2003

9. Hott JS, Henn JS, Sonntag VK: A new table-fixed retractor for anterior odontoid screw fixation. Technical note. J Neurosurg (Spine 3) 98:294-296, 2003

10. Palmer S: Use of a tubular retractor system in microscopic lumbar discectomy: 1-year prospective results in 135 patients. Neurosurg Focus 13 (2): Article 5, 2002

11. Puttlitz CM, Goel VK, Clark CR, et al: Pathomechanisms of failures of the odontoid. Spine 25:2868-2876, 2000

12. Lennarson PJ, Mostafavi H, Traynelis VC, et al: Management of type II dens fractures: a case-control study. Spine 25: 1234-1237, 2000

13. McLain RF: Salvage of a malpositioned anterior odontoid screw. Spine 26:2381-2384, 2001

14. Sasso RC: C2 dens fractures: treatment options. J Spinal Disord 14:455-463, 2001

15. Subach BR, Morone MA, Haid RW Jr: Management of acute odontoid fractures with single-screw anterior fixation. Neurosurgery 45:812-820, 1999

16. Vishteh AG, Baskin JJ, Sonntag VKH: Techniques of cervical discectomy with and without fusion. Oper Techn Neurosurg 1:84-89, 1998

17. Ziai WC, Hurlbert RJ: A six year review of odontoid fractures: the emerging role of surgical intervention. Can J Neurol Sci 27:297-301, 2000

Manuscript received November 17, 2003.

Accepted in final form December 22, 2003.

Address reprint requests to: Allen B. Kantrowitz, M.D., Neurosurgery Associates of Miami Beach, 21110 Biscayne Boulevard, Suite 106, Aventura, Florida 33180. email: abkns@aol.com. 